\title{
EITORIAL
}

\section{Armut, Reichtum und Sozialstaat}

\author{
Claus Schäfer, Claudia Bogedan \\ Till Müller-Schoell
}

\begin{abstract}
Die soziale Ungleichheit in Deutschland wächst. Wesentliche Treiber dieser Entwicklung sind die anhaltend schlechte Arbeitsmarktlage und fehlende wie auch falsche sozial- und verteilungspolitische Maßnahmen: So wurde unter anderem der Schutz bei Arbeitslosigkeit reduziert. Gleichzeitig aber hat die Zahl prekär entlohnter Beschäftigungsverhältnisse zugenommen, was immer mehr „Armut trotz Arbeit" erzeugt. Seit Einführung von Hartz IV hat sich die Anzahl der sogenannten Aufstocker, also der Menschen, die trotz Erwerbstätigkeit Leistungen der Grundsicherung beziehen, mehr als verdoppelt. Diese Gegenwartsprobleme werden nicht nur absehbar andauern; sie werden in Zukunft sogar mit neuen Verteilungsproblemen kumulieren. Denn die Veränderungen im Erwerbssystem bergen in Kombination mit den Einschnitten im Rentensystem ein hohes Armutsrisiko für künftige Rentnergenerationen - zumal nur gering wachsende, stagnierende oder gar rückläufige Einkommen immer mehr Menschen die Möglichkeit entziehen, Kürzungen in den öffentlichen Sozialleistungen durch private Vorsorge auszugleichen. Erwerbslose haben diese Chance ohnehin nicht. Außerdem geht mit der wachsenden Einkommensungleichheit eine ungleiche Teilhabe an Lebenschancen einher - mit absehbaren ökonomischen und gesellschaftlichen Folgelasten. Exemplarisch dafür ist die Kinderarmut. Sie birgt für die große Gruppe von Kindern, die in Armut leben oder zeitweise gelebt haben, die Gefahr, dass sie wegen armutsbedingter Defizite aus ihrem unmittelbaren Umfeld wie auch gesellschaftlich ausgeschlossenen werden. Dass Einkommensarmut, in der Kinder leben, auch bereits die Weichenstellung für ihren späteren Ausschluss vom Arbeitsmarkt bedeuten kann, ist Teil des Teufelskreises der Armut.
\end{abstract}

Hat der Ausbau des Sozialstaats nach 1950 - trotz vorhandener Schwachpunkte - die soziale Ungleichheit noch spürbar gemindert, so kann der Sozialstaat heutiger Form notwendige Schutzfunktionen nicht mehr angemessen ausfüllen oder bekommt sie sogar entzogen. Alte soziale Risiken kehren zurück, neue werden nicht adäquat abgesichert. Der Rückbau bisheriger Sozialstandards erfolgt in der vagen Hoffnung auf eine dynamische ökonomische Entwicklung. Jedoch: Das Wachstum bleibt seit Jahren niedrig, die Arbeitslosigkeit weiterhin hoch, die öffentlichen und privaten Investitionen sinken. Umso schwerwiegender sind die negativen Nebenwirkungen: Die Entlastungen in Form von einseitigen Steuersenkungen und gedeckelten Sozialabgaben hinterlassen "klamme“ Haushaltskassen. Das wiederum verengt den Spielraum für sozialpolitische Gestaltung. Sinkende Sozialleistungen und reduzierte Angebote an öffentlichen Gütern, vor allem in Infrastruktur, Bildung und allgemeiner Daseinsvorsorge, sind die Konsequenz.

Dass trotz der beobachtbaren Fehlentwicklungen ein nachdrückliches politisches Gegensteuern ausbleibt, war Anlass, das WSI-

Herbstforum Ende 2007 unter den Titel „Armut, Reichtum und So- zialstaat - Ist die soziale Spaltung noch lösbar?" zu stellen. Die dort gehaltenen Vorträge sind die Grundlage für dieses Schwerpunktheft. Die Beiträge konzentrieren sich auf die Formen der Einkommensarmut: Armut im Alter, Familien- und Kinderarmut sowie Armut trotz Arbeit. Sie verweisen zudem auf die europäische Dimension von Armutslagen und Armutspolitiken. Auch der Blick auf die Elitenstruktur bzw. den Reichtum hilft bei der Suche nach den Ursachen ungerechter Verteilung wie nach Lösungsmöglichkeiten. Er schärft die Erkenntnis, wie fundamental soziale Durchlässigkeit, politische Teilhabe, organisierte Konfliktfähigkeit und schließlich politischer Gestaltungswille für eine gerechte, sozial ausgewogene Gesellschaft sind. In unserem Nachbarland Österreich steht Armutsbekämpfung zumindest ausdrücklich auf der Agenda der Regierungspolitik. Eine für 2009 geplante armutsfestere Grundsicherung soll die auch in Österreich vorhandene Schere zwischen der vorhandenen Armut und der sozialstaatlich bekämpften Armut durch höhere Sozialleistungen schließen.

Dieses Beispiel sollte auch für Deutschland ein Impuls sein, Reformen anzugehen, die die soziale wie auch die ökonomische Lage nachhaltig verbessern. Dazu gehört in erster Linie ein politischer $\mathrm{Pa}$ radigmenwechsel: weg von der falschen Alternative zwischen „Sozialem" und „Ökonomischem", was allzu oft auf eine Umverteilung von unten nach oben und anschließende Wachstumsschwäche hinauslief. Der Gegenentwurf ist eine gerechtigkeitsorientierte Politik, die zum einen Armutsursachen aktiv entgegenwirkt und zum anderen versucht, die Armutsfolgen bisheriger Politik abzufedern. Die Erhöhung monetärer Transferleistungen zur Bekämpfung der Einkommensarmut wäre ein notwendiger erster Reformschritt. Hinzukommen müssen Investitionen in bessere Lebenschancen - allen voran Bildungs-, Erwerbs- und Aufstiegschancen -, die sowohl wachstumsfördernd wirken als auch die soziale Transparenz und demokratische Qualität einer lebenswerten Gesellschaft ausmachen.

\footnotetext{
Dr. Claus Schäfer, Wissenschaftler im WSI in der Hans-BöcklerStiftung. Arbeitsschwerpunkte: Verteilungsanalyse von Einkommen und Lebenslagen. e-mail: Claus-Schaefer@boeckler.de Claudia Bogedan, Wissenschaftlerin im WSI in der Hans-BöcklerStiftung. Arbeitsschwerpunkte: Sozial- und Arbeitsmarktpolitik, vergleichende Wohlfahrtsforschung e-mail: Claudia-Bogedan@boeckler.de Dr. Till Müller-Schoell, Wissenschaftler im WSI in der Hans-BöcklerStiftung. Arbeitsschwerpunkte bis 1.2.08: Arbeitsmarktpolitik, verg/eichende Wohfahrtsstaatsforschung e-mail: Till-Mueller-Schoel/@boeckler.de
} 